\title{
An Information Architecture Framework for the Internet of Things
}

\author{
Flávia Lacerda ${ }^{1}$ (D) Mamede Lima-Marques $^{2}$ (D) $\cdot$ Andrea Resmini $^{3}$ (D)
}

Received: 6 December 2017 / Accepted: 30 October 2018 / Published online: 14 December 2018

(C) The Author(s) 2018

\begin{abstract}
This paper formalizes an approach to the Internet of Things as a socio-technical system of systems and a part of the infosphere. It introduces a principle-based, human-centered approach to designing Internet of Things artifacts as elements of contextual cross-channel ecosystems. It connects the Internet of Things to the conceptualization of cross-channel ecosystems from current information architecture theory and practice, positing that the Internet of Things is both a formal, objective superset of any given ecosystem and a contextual, subjective subset of specifically instantiated ecosystems. The paper argues for the necessity of a transdisciplinary theoretical framework to promote a human-centered generative understanding of the Internet of Things phenomena and their consequences, in accordance with the Metamodel Methodology (M3). It proposes a phenomenologygrounded information architecture model detailing a set of 16 principles and secondary heuristics grouped according to an architectural perspective, which identifies guidelines that support the design of Internet of Things artifacts considering their objective characteristics; a human perspective, which identifies guidelines that support the design of Internet of Things artifacts considering subject/ object relationships and the production of meaning; and a systemic perspective, which identifies guidelines that support the design of Internet of Things artifacts as relational parts of information-based ecosystems. These principles and guidelines are meant to provide the foundations for a practice-based approach to designing the Internet of Things-enabled information ecosystems.
\end{abstract}

Keywords Information architecture - Internet of things · Information ecosystems · Infosphere $\cdot$ Cross-channel

Flávia Lacerda

flavialacerdaoliveira@gmail.com

Andrea Resmini

andrea.resmini@ju.se

Extended author information available on the last page of the article 


\section{Introduction}

As convergence between physical and digital increases (Conti et al. 2012), information connects previously independent information spaces into ecosystems that participate in a larger "infosphere" (Floridi 2010) tying together people, artifacts, and places. This infosphere also includes the so-called Internet of Things. The term was introduced at the end of the 1990s, possibly by Ashton (2009), and indicates the growing worldwide network of objects that can react to their environment and share data remotely. Artifacts, a part of the Internet of Things, are called "smart objects" and are technically simple embedded systems provided with sensors, actuators, and networking capabilities (Kopetz 2011).

It is worthy of note that while data exchanges usually happen through the internet, the Internet of Things is currently framed as a separate interoperating entity. This connection between physical artifacts that do not share the same location allows systems "to access remote sensor data and to control the physical world from a distance," giving rise to "new synergistic services that go beyond the services that can be provided by an isolated embedded system" (Kopetz 2011, p. 307).

In the Internet of Things, commonplace artifacts such as umbrellas, mirrors, cars, or prostheses, assume agency. Embedded with computational power and networking capabilities, they become capable of receiving, transmitting, and producing information (Rose 2014) independently of direct human intervention (Mitew 2008). Sensors perceive stimuli from the environment; microprocessors, either local or remote, elaborate them; actuators modify the current setup; Wi-Fi chips allow constant communication with remote data processing facilities or other artifacts, and interfaces modulate interactions with human beings. An object's agency is here understood in accordance with Latour's Actor-Network Theory (Latour 1996) and its extension of decisional and functional capabilities to non-human entities, actors, or actants.

Research focusing on the technological aspects of the Internet of Things is essential, but clearly not sufficient. Major issues concerning data safety, privacy, awareness, and informed consent are inextricably entwined with the increasing pervasiveness of the resulting blended spaces (Benyon 2014) in which smart objects are entrusted with a degree of autonomy and decision-making prerogatives. The behavior of smart objects is after all "contingent on the decisions made by designers, regulators, and markets of empowered buyers" (Greenfield 2006, p. 171) and their long-term ethical and political impact on the lives of individuals, on organizations, and on governments depends on the complex interplay of these decisions.

\section{The Internet of Things and the Infosphere}

The concept of the "infosphere" was introduced by Sheppard (1971) and further developed by Toffler (1980, p. 172). Toffler argued that after the agricultural age and the industrial age, we were entering a new information age characterized by the radical alteration of the existing infosphere: "the emerging Third Wave infosphere makes that of the Second Wave era-dominated by its mass media, the post office, and the telephone - seem hopelessly primitive by contrast." This transformation was the consequence of the addition of "a whole new strata of communication to the social 
system," with the resulting infosphere being characterized by Toffler as the expression of a decentralized, demassified, bottom-up post-industrial society in which the Internet plays a central role and where value equates with information, not products.

Floridi (2010) has more recently repurposed the concept from the perspective of the philosophy of information, defining the infosphere as a complex "environment constituted by all informational entities (and) thus including informational agents as well, their properties, interactions, processes, and mutual relations." Floridi's infosphere also clearly extends beyond the confines of what is digital and what is human to include all types of environmental information and all types of softwarebased agents, those powering social media algorithms and those driving autonomous cars. Floridi (2014, p. ix) also clearly identifies in design the primary way to counterbalance technological pushes in the infosphere: design is not limited to the simple production of value but plays a larger role in the building of "an information ethics for the whole infosphere and all its components and inhabitants," including the "artificial, digital, or synthetic."

Following Floridi, rather than a network of individual points, we consider the Internet of Things a part of the infosphere and a semantic environment (Hinton 2014, p. 212). Ashton (2009) originally introduced the idea that individual physical objects could be connected with one another and to the Internet, be able to record and exchange real-world data, and thus act in the world independently of people, overcoming limitations of time and attention. This vision of a secondary, separated layer of connected objects presents a thoroughly different angle in the work of Mark Weiser (1999). Weiser describes "ubiquitous computing" as the embedding of informationprocessing capabilities into objects and environments and a move towards a "calm(er) technology," that is, a technology that disappears in the background of commonplace activities and largely imperceptible to everyday use.

Weiser's systemic description echoes those of McLuhan (1965, p.71), that "(i)n this electric age we see ourselves being translated more and more into the form of information (...). By means of electric media, we set up a dynamic by which all previous technologies that are mere extensions of hands and feet and bodily heatcontrols - all such extensions of our bodies, including cities - will be translated into information systems," and Mitchell (2006, p.1), who observed how "our cities are fast transforming into artificial ecosystems of interconnected, interdependent intelligent digital organisms."

We posit that this framing also closes a conceptual loop: the Internet provided the socio-technical basis for identifying in the network the organizational form of the late twentieth and early twenty-first centuries (Castells 2002). By addressing the Internet of Things as part of the infosphere we logically recompose the picture by formally bridging the rift between what is digital and the physical world of objects and spaces: anything that can be connected will be connected and will become a "smart" object (Kuniavsky 2010) acting in the world.

In terms of its ontology (Benyon 2014, p. 80), the Internet of Things model dramatically flips the table on canonical information systems approaches. Instead of a handful of very powerful "intelligent" devices, be these mainframes, notebooks, or even multi-purpose smartphones, it postulates billions of computationally weak objects specifically designed and engineered to accomplish one single task, sense the temperature, measure an increase or decrease in the amount of 
light, but that can leverage the computational and informational capacity distributed throughout the network (Rose 2014).

In terms of its topology (Benyon 2014, p. 80), the Internet of Things can be considered a system of systems interconnected at different scales. It variously intersects with both the infosphere, of which it is a subset, and with the information architecture conceptualization of cross-channel ecosystems (Resmini and Lacerda 2016), of which it configures both an objective, formal superset, and a subjective, contextual subset. It is an objective superset of this ecosystem model because it is, by definition, the system of all systems in which "smart objects" participate. As of early 2017, Gartner estimated it could comprise as many as 8.4 billion devices (Gartner 2017). It is also a subjective subset as, through subject/object relationships, certain specific Internet of Things artifacts become part of contextual, vastly smaller ecosystems that also contain biological, spatial, and urban components that are kept together by means of an information layer. These information-based ecosystems are what Resmini and Rosati call a "ubiquitous ecology" (2009) and Resmini and Lacerda (2016) have more precisely defined as cross-channel ecosystems.

\section{Information Architecture}

Information architecture is a field of study and a practice that deals with the structural design of information spaces. Pioneered by the works of architect Richard Saul Wurman in the 1970s and the 1990s (Wurman 1997), information architecture became a mainstream phenomenon in connection with the growth of the World Wide Web and the subsequent publication of Rosenfeld and Morville's "Information Architecture for the World Wide Web" (Rosenfeld and Morville 1998). Variously defined through the years as first "the building of information structures that allow others to understand" (Wurman 1997, p. 17), "the structural design of shared information environments" (Rosenfeld and Morville 2006, p. 4), and more recently as "the practice of deciding how to arrange the parts of something to be understandable" (Information Architecture Institute 2016), information architecture was initially represented as a practice mostly concerned with specific digital artifacts such as websites, in accordance with Rosenfeld and Morville's original definition of it as a way for "understanding and conveying the big picture of a web site" (1998, p. 14). Practicing information architects "chose to define the discipline through the artifacts of the practice" (Resmini 2013), a reductive view of the field considerably distant from Wurman's original approach.

More recently, information architecture has been thoroughly redefined. This reframing originates with Resmini and Rosati (2011) who introduced the concept of pervasive information architecture as the medium-aspecific design of digital/physical information spaces that span multiple locations, products, and services, emphasizing the role of placemaking in the process $(2011$, p. 63). They argue that the role of information architecture is and has always been that of structuring "the process" (Resmini and Rosati 2011, p. 52) rather than producing concrete, individual artifacts. Resmini (2013) clarified this concept arguing that the main outcome of "information architecture, unlike other fields of design, is abstract: it is (...) 'sense-making' - the arrangement and organization of the information structure" and that framing a discipline through the incidental artifacts it produces was an epistemological mistake. 
Mainstream adoption in the practice has followed, reflected in industry publications (Tate 2011; Norris 2013), conferences, in more reflective, theoretical contributions (Lucas et al. 2012; Hinton 2014), and in academic discourse (Resmini 2014; Benyon and Resmini 2017).

The current understanding of information architecture posits that "the illusion of the web as a library" (Resmini 2014, p. v) has been superseded. Conversely, so has the idea that library and information science is the only relevant body of knowledge for the discipline. This has opened the field to contributions coming from disciplines such as architecture, cognitive science, and systems thinking, among others, and has led to conceive the Internet not as "a different and separated world" or domain, but rather as a part of "a larger mechanism where consumption and production of information happen across multiple contexts through multiple devices" that easily "blend digital and physical" (ibidem).

As a response, both conceptually and in the practice, the "information spaces" structured by the field have extended their reach: information architecture has been moving "away from the single artifact, the website, to consider the entire product or service ecosystem as a complex, cross-channel information-based beast, some parts of which might not be online or might not even be digital at all" (ibidem).

Resmini and Lacerda (2016) have been more recently and more precisely defining these information spaces as cross-channel ecosystems, "actor-driven, informationbased, semantic constructs connecting individual touchpoints into (a) transient architecture" configuring a blended, digital/physical space of action (Benyon and Resmini 2017). Actors, human, and non-human agents navigate the space of the ecosystem traversing the informational seams that connect touchpoints in order to achieve a desired future state.

From an information architecture viewpoint, the Internet of Things fully realizes the reframing tenet that digital information is pervasive. This paper argues that crosschannel ecosystems are local, contextual instantiations of Floridi's infosphere bounded by the experiences currently being observed or investigated. In this view, the Internet of Things is also a different, broader subset or instance of the infosphere, as it is ontologically framed as the part of it populated by "smart" artifacts. It follows that it also logically constitutes a possible part of any local or contextualized information ecosystem.

\section{Principle-Based Approaches to Design}

Considering the focus on emplaced experience, we adopt here a phenomenological approach to information architecture (Lima-Marques 2011). Phenomenology (Husserl 1931) offers a methodologically precise framing to investigate the experiential and systemic nature of subject-object-knowledge relations (Tuan 1977; Norberg-Schulz 1971), and consequently, the interaction between humans (as cognitive systems) and spaces/objects (also systems). The article also adopts Van Gigch and Pipino's Metamodel Methodology (M3) to distinguish and analyze the epistemological, scientific, and practical aspects of information architecture (Van Gigch and Pipino 1986; Lacerda and Lima-Marques 2014; Lacerda 2015) as they relate to the design of information-based ecosystems that comprise the Internet of Things instantiations. 
The M3 (Fig. 1) represents the dynamics of any field of knowledge through a metamodel comprising three interconnected layers: a lower layer of "solutions," where the practice operates, whose inputs are practical problems and whose outputs are solutions to those problems (practice); a middle "theory" layer, whose inputs are scientific problems and whose outputs are theories and models (science); and a "paradigmatic" top layer, whose inputs are philosophical questions and whose outputs are changes to the current paradigm (epistemology). Consolidated changes affect the practice and impact the theories and models that a discipline adopts to solve its problems. In the long run, these changes contribute to posing new or different philosophical issues that challenge its fundamental premises. An opposite flow runs from the paradigm layer to the solutions layer as the practice also redefines itself in the light of new interpretations of the field itself.

This M3-based approach conceptually addresses the existing gap Floridi individuates in Western culture between "episteme (science and 'knowledge that'), which is highly valued and respected, and techne (technology and 'knowledge how'), which is seen as secondary" (Floridi 2014, p. 85) by making them part of a systemic process.

We posit that while information architecture has maintained its epistemological purpose as the structural design of information spaces that support an agent's production of meaning, it has nonetheless seen its top layer change under the influence of socio-technical changes (Kirby 2009; Pepperel and Punt 2000) that have redefined what these spaces are. Such changes are yet to be fully addressed in the science and practice layers (Resmini 2013), even though these spaces are now experienced differently, used differently, and have a substantially more vast impact on the society as a whole.

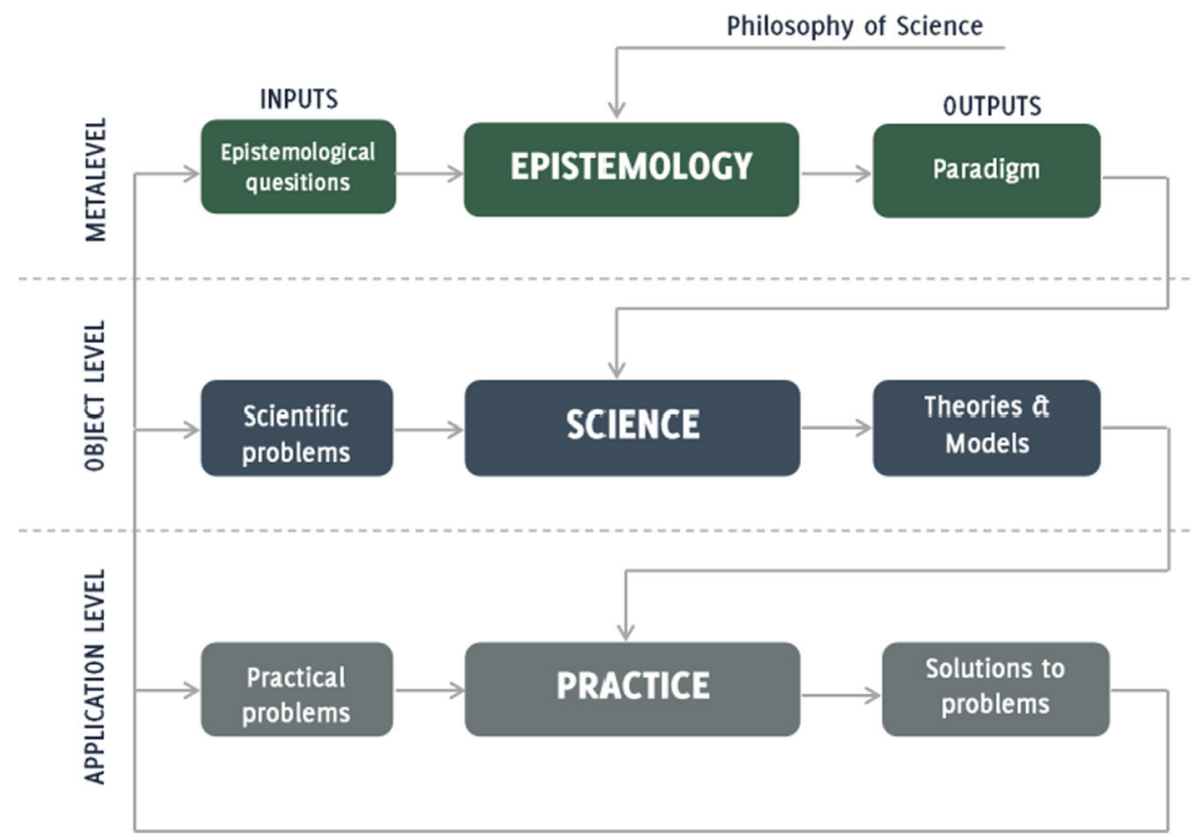

Fig. 1 The M3 Metamodel Methodology (Van Gigch and Pipino 1986. Adapted by Lacerda and LimaMarques 2014) 
The Internet of Things, as a part of the infosphere, is a manifestation of these new information spaces. It materializes a world where information is pervasive, embedded in billions of interconnected smart things, each one a system part of larger ecosystems. To support reflective practices in information architecture so that a body of scientific knowledge current with the current socio-technical needs and wants may emerge in the science layer and ultimately lead to challenging the current understanding of the discipline in the epistemology layer, we propose a principle-based approach to the design of Internet of Things-enabled information ecosystems.

Principle-based approaches to design have a long tradition that can be traced all the way back to Roman architecture and Vitruvius' "Ten Books on Architecture" (1999), written in the first century BC. In respect to the current practices of design, principlebased approaches carry specific merits that descend from their own limitations: principles are intended to be broad generalizations and, as such, they have a degree of aspecificity and medium-independence that makes them extremely suitable to the design of complex environments such as those emerging from the collision of digital and physical. When Vitruvius lists firmitas, utilitas, and venustas (solidity, usefulness, beauty) as the three core characteristics of good architecture, he is providing us with meta-descriptors which we can still adopt and use today. A mobile app can and should be robust, useful, and esthetically pleasing: its robustness will be designed and interpreted differently from that of the Domus Aurea, but the principle holds, as the app needs to work efficiently and not break down during use.

This long-lasting applicability of principles is recognized in the literature. Ishii (2012) observes that innovation should be driven by a vision supported by foundational concepts and principles, noting that, proportionally, technologies have an estimated lifespan of 1 year, user needs remain valid for around 10 years, while principles could last for more than a hundred years. Greenfield (2006, p. 257) elaborates on the aspecificity of principles and links them to guidelines, maintaining that "principles are necessary, but not sufficient: they constitute not an end, but a beginning." Greenfield considers guidelines as clearly useful to drive design, even though limited by their being contingent, temporary, and incomplete. The abstraction of concepts in the form of a model is always intended to be a useful fiction adopted to facilitate understanding and comparison, and ultimately turn these into action and design.

More specifically considering the design of information-based environments, Lucas et al. (2012, p. 140) state that "if pervasive computing arrives without adequate principles in place to guide it, it will quickly result in incoherent, unmanageable, malignant complexity." Resmini and Rosati (2009) suggest that "since information architecture relies on principles largely independent from any specific medium or practice, it provides a flexible but solid conceptual model for the design of crosscontext interaction models." They propose a heuristic approach based on information architecture-derived, medium-independent indicators (Resmini and Rosati 2011).

Foltz (1998) observes that the utility of a principle arises from its ability to help to solve a wide class of problems and lists a set of properties that define effective design principles: (a) sufficient, as opposed to necessary, may be one way of many to meet a requirement; (b) proven in practice; (c) specific enough to be applicable covering a significant class of problems; and (d) consequential, producing real impacts for the design. Building on this set of properties, Foltz suggests a general scheme for presenting design principles which we adapt here as a general descriptive structure for our set 
of design propositions, and consisting of (a) principle statement, (b) principle description, and (c) heuristics, methodological directions for application derived from previous experiences with similar problems.

\section{An Information Architecture Framework for the Internet of Things}

Considering the paradigm shift we described (epistemology layer), we propose an information architecture framework (science layer) based on 16 principles and their related heuristics that should guide the design of artifacts in the Internet of Things (practice layer). The framework is multidisciplinary at its core and relies on theories and models from the field of information architecture while borrowing elements from disciplines such as architecture, cognitive science, systems thinking, and philosophy. The framework adopts a phenomenological approach centered on human-object experience (Husserl 1931) to properly address the nature of interactions between humans, spaces, and artifacts in systemic terms (Tuan 1977; Norberg-Schulz 1971; LimaMarques 2011).

The 16 principles are meant to be applied systemically and are grouped in three broad categories: architectural principles, which consider the perspective of information spaces and artifacts; human principles, which consider the subjective perspective of subject/object interactions; and systemic principles, which consider the relational perspective between artifacts, actors, and systems in information ecosystems.

Figure 2 illustrates the three groups of principles and their relationships as overlapping spheres, with the goal of providing a visual representation of the overall information architecture model that illustrates its pervasive structure.

The central sphere (in light green) shows the subjective perspective of human experience representing a human-centric approach. This layer illustrates phenomenological space, or place (Tuan 1977; Norberg-Schulz 1971), as it emerges from the interactions between subjects and objects. Human principles and heuristics apply to this layer.

The second sphere (in light blue) represents the topological space of artifacts: here is where interactions between subjects and objects occur and where the principles and heuristics of the architectural perspective apply.

The third sphere (in light red), which embraces the other two indicating their connection, shows the systemic characteristics that define the elements forming the information ecosystem, of which include the subject and objects as systems, the ecosystem of which they are part, and their semantic relationships. Systemic principles and heuristics apply to this perspective.

"Internet of Things artifact" is used all throughout the list of principles to broadly address both objects and spaces augmented or transformed by the presence of "smart objects." It is also worth mentioning that while specifically aimed at Internet of Things artifacts, the principles might also find application in the design of cross-channel ecosystems.

\subsection{Human Principles}

Principles belonging to the human perspective identify guidelines that support the design of Internet of Things artifacts considering their subjective characteristics, the 


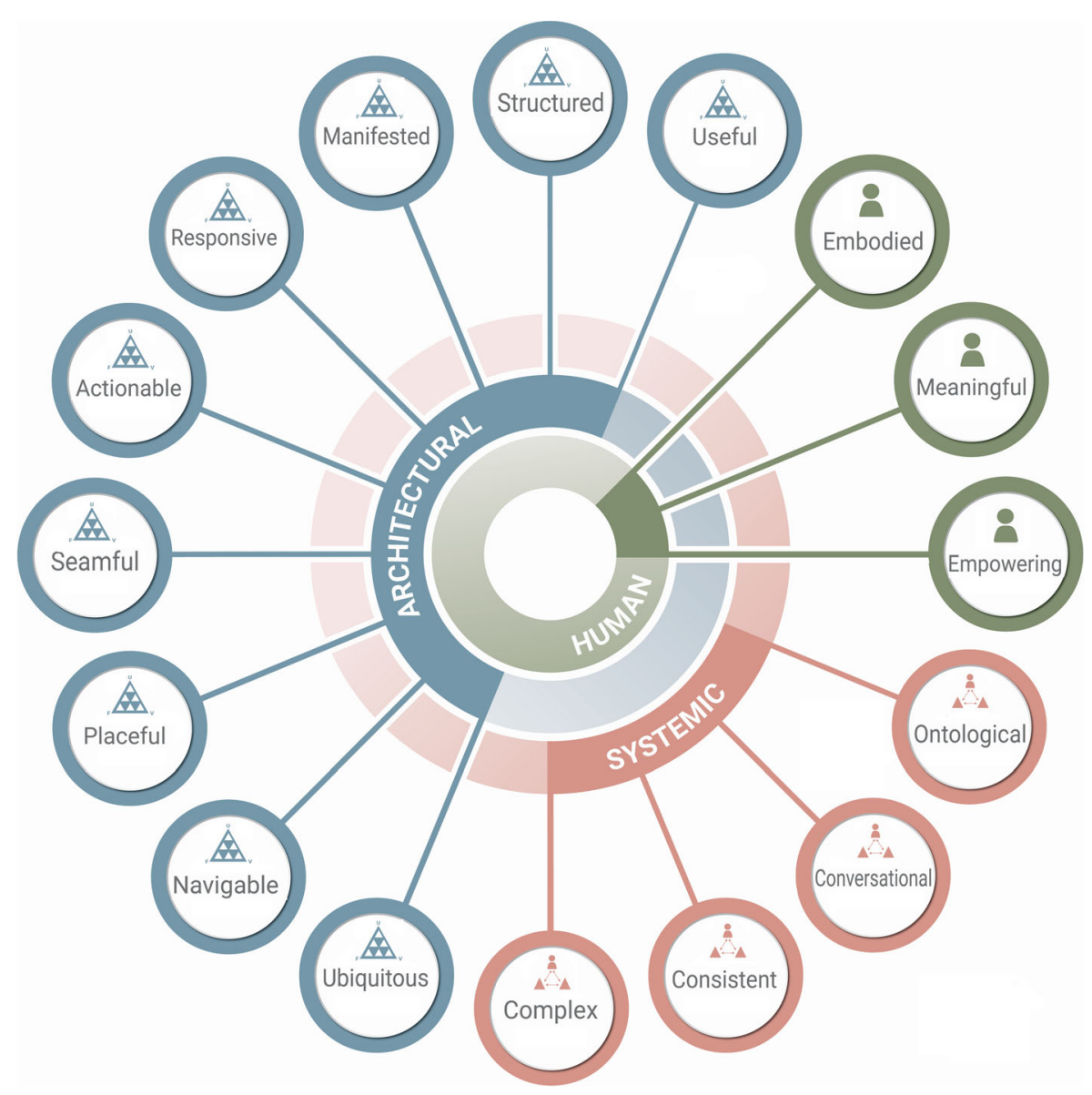

Fig. 2 A visual representation of the framework (Infographics: M. Alvim.)

emergence of meaning, and the potentiality for action through subject/object relationships.

\subsubsection{Embodied}

Human cognition is embodied, context-situated, and action-oriented "Cognition depends on the kinds of experiences that come from having a body with particular perceptual and motor abilities that are inseparably linked" (Thelen et al. 2001). Human sense-making is an embodied process emerging from the interaction of a specific body with its environment considered as a biological, psychological, and cultural context (Varela et al. 1993).

Heuristics Design Internet of Things artifacts to support embodied and situated interactions. Consider information can be conveyed bodily through auditory, visual, tactile clues, through smell and taste, or through whole-body experiences such as orientation, directionality, or weight. 


\subsubsection{Meaningful}

Humans attribute meaning to interactions with artifacts Phenomenologically, meaning is produced through the relationships that emerge between an artifact, its context, and an agent. In design terms, it is the result of the intentions of the designers (intended meaning), the affordances of the system (constructed meaning), and the conceptualization of actors (received or reconstructed meaning) (Kazmierczak 2003; Dourish 2004).

Heuristics Design Internet of Things artifacts focusing primarily on received meaning and on the way the artifacts themselves help actors reconstruct intended meaning. Consider how meaning is created through the interplay of the different elements. Allow space for the production of unintended meanings that might increment the resilience of the system. Carefully establish the relationship between value production and constructed meaning.

\subsubsection{Empowering}

Meaningful artifacts expand one's potentialities of action Technology extends and expands human capabilities. It allows actors to accomplish tasks they could not accomplish otherwise. Successful artifacts separate from the environment and become an extension of the actor's body (Dourish 2004).

Heuristics Design Internet of Things artifacts with the goal of making everyday life easier, more efficient, and more enjoyable. An artifact's architecture should be visible and actionable to allow for a degree of control, and actor's informed and empowered consent to use.

\subsection{Architectural Principles}

Principles belonging to the architectural perspective identify guidelines that support the design of Internet of Things artifacts considering their objective characteristics. The principles share the premise that every artifact or space has an ontological nature since its existence is independent of individual subjects (Tuan 1977), and that they all possess an underlying architecture that is also an information architecture.

\subsubsection{Useful}

Artifacts must be useful Design Internet of Things artifacts with the goal of providing value to actors. Artifacts should balance usefulness to individuals with overall social usefulness.

Heuristics Design Internet of Things artifacts with the purpose of establishing grounds for a meaningful subject-object relationships. Follow the principle of least effort: considering any activity will only be performed if its benefits are equal to or greater than its costs, 
including operational costs; meet an actor's basic needs before satisfying any additional ones.

\subsubsection{Structured}

Artifacts are instantiated structurally The structure of an Internet of Things artifact comprises all aspects of its technological and physical architecture.

Heuristics Design Internet of Things artifacts structurally to allow for a larger degree of medium independence. Consider their internal architecture and how it responds to constraints, opportunities, limitations, and infrastructural issues such as confidentiality, integrity, availability, reuse, interoperability, and sustainability.

\subsubsection{Manifested}

Artifacts are esthetically manifested Esthetics is understood here as a "manifestation," relative to what is perceived by the subject. In terms of information architecture, this is represented by the harmonious coming together of the parts: the juxtaposition of structural elements in space represents the identity of the design through its composition or configuration.

Heuristics Consider spatial primitives (Norberg-Schulz 1971) such as proximity, enclosure, and sequence. Consider how proportion, harmony, symmetry and asymmetry, contrast, and organization alter one's understanding of the way the artifact works. Design information architectures that minimize cognitive load through progressive disclosure, organization and categorization (sort, label, integrate, prioritize), and grouping (shrink, hide, aggregate) (Maeda 2006; Lidwell et al. 2010).

\subsubsection{Responsive}

Artifacts must respond effectively, reliably, and safely to people's needs Internet of Things artifacts have an agency. They respond to the stimuli of the environment, communicate among themselves, take decisions, and act with varying degrees of autonomy. They should do it in such a way that they are effective, reliable, and safe (McCullough 2004; Norman 2009).

Heuristics Design Internet of Things artifacts considering criteria such as accessibility, portability, contingency, empathy, and predictability. Artifacts should be accessible and so should the information or content they convey. Artifacts should support calm interactions, not be demanding, and allow for actor control: they should not try to force decisions on actors nor act unpredictably. Actors should both control and be informed precisely of the amount and type of decisions that they delegate to artifacts. Failsafe mechanisms should be in place for actions that might produce unwanted or irreversible changes. Safety considerations should include both physical and psychological aspects. 


\subsubsection{Actionable}

Artifacts should be actionable Affordances are the possibilities of (inter)action that arise between an actor and an artifact because of its perceivable properties (Gibson 1979). Phenomenologically, as they are relational opportunities that emerge through the coupling of the abilities of the body and the characteristics of the world, they allow multiple interpretations and ones which might go beyond intended functionality: "the properties of the object and the intentions of the subject (...) are not only intermingled; they also constitute a new whole" (Merleau-Ponty 1963, p. 13).

Heuristics Artifacts should communicate their capabilities and the opportunities for the action they allow and should allow unplanned for or unforeseen use emerging from the context of use, the properties of the environment, or the history and motivations of actors.

\subsubsection{Seamful}

Artifacts should have perceptible seams allowing unobstructed movement Experiences should flow unobstructed but seams should be felt when necessary and exploited to provide navigational clarity to the ongoing process (Chalmers et al. 2004). Seams play a vital role in the information architecture of an ecosystem, as they "allow the passage of content and information from touchpoint to touchpoint and across channels, and enable actors to progress in their individual experience" (Resmini and Lacerda 2016). The principles of calm technology (Weiser 1999) apply.

Heuristics Design Internet of Things artifacts so that the transitions between them flow naturally and do not obstruct action, but make seams perceptible when the actor's attention is required. Present possible multiple points of departure clearly to avoid confusion and uncertainty and show progress accordingly.

\subsubsection{Placeful}

Meaningful artifacts become places Placemaking reflects a conscious arrangement of elements to create environments that accommodate activities and interactions in a meaningful way (Harrison and Dourish 1996) and thus become "places," "pause(s) in movement. Animals, including human beings, pause at a locality because it satisfies certain biological needs. The pause makes it possible for a locality to become a center of felt value" (Tuan 1977, p.138). Place anchors experiences: "the variance of context and the capability to act in blended space from a number of locations makes the creation of a sense of place an important component of design" (Benyon and Resmini 2017).

Heuristics Design Internet of Things artifacts so that they allow pausing. Introduce a degree of manipulability to support appropriation, including naming. Support the artifact's individuality and clearly separate it from its environment, but gives it a meaningful, identifiable role in the larger context of its ecosystem. Consider permanence of context and object/subject memory as a way to support the consolidation of emergent relationships. 


\subsubsection{Navigable}

Artifacts should be navigable People use the spatial and environmental information to meaningfully move through space. Both subjective factors deriving from previous experience, language, culture, education, or age, and objective factors, such as the structural elements allowing or preventing sight or passage that are part of the navigable space itself are used in wayfinding behavior (Lynch 1960; Arthur and Passini 1992) and to help one's sense of orientation and directionality in a physical and digital space.

Heuristics Design Internet of Things artifacts so that the paths between the elements of an actor's ecosystem are navigable. Consider the information architecture of individual artifacts as a piece of the larger information architecture of the contextual ecosystem, and consider objects spatially: "place is a special kind of object" (Tuan 1977, p. 12). Inform people of where they are and the possible paths they can take. Provide views at different scales and implement unequivocal and systemically coherent navigation clues. Use landmark elements to suggest further possibilities. Consider physical or psychological distance as a parameter for separating logically separated activities. Provide alternative views and design them coherently. Provide signals at decision points and when traversing seams.

\subsubsection{Ubiquitous}

Artifacts are connected to the infosphere and act both locally and globally Internet of Things artifacts store and process information. Their presence is simultaneously physical and local, digital and global. They transform spaces into nodes part of the infosphere and potentially turn any local, transient, synchronous event into networked, persistent, asynchronously retrievable data.

Heuristics Design Internet of Things artifacts considering their physical/digital context: these objects are very often hyperlocal, for example, a thermostat keeping track of the temperature of a specific room, but in a potential conversation with the whole of the infosphere. Ethical, political, and legal concerns for archiving, mining, interpreting, and sharing the data being captured or generated should be a major concern. Consider transparency in respect to what data is being collected, what will be done with it (McEwen and Cassimally 2013), and the long-term perspectives of continuous use. Carefully evaluate the transformative impact of embedding computing and information processing into personal or social spaces.

\subsection{Systemic Principles}

Principles belonging to the systemic perspective identify guidelines that support the design of Internet of Things artifacts by considering them as part of the infosphere and of contextual information-based ecosystems. These principles address dynamics and behavior as a result of structure (von Bertalanffy 1998; Meadows 2008) and consider 
how information flows through artifacts, how actors and artifacts interact systemically, and with what consequences.

\subsubsection{Complex}

Artifacts Connect to Produce Complex and Emergent Ecosystems The Internet of Things is a system of interconnected systems participating in the infosphere. From an experience design and information architecture perspective, subsets of the Internet of Things are also part of contextual information-based, cross-channel ecosystems. While individual artifacts possess structure, manifesting itself esthetically and serving the artifact's purpose, the overall structure of the ecosystem is an emergent architecture depending both on the individual artifacts' characteristics and on the subject/object relationships that are at the root of an actor's choices in the instantiation of a specific contextual cross-channel ecosystem.

Heuristics Design Internet of Things artifacts considering how they will behave as part of the dynamic set of elements that combine to form a contextual ecosystem in which actors can have meaningful experiences. Identify an artifact's semantic and spatial boundaries and how it communicates with actors and other artifacts. Standardize or design according to basic modular structures that can be repeated. Consider the problems that any encounter between complex technical systems and the people using them brings about and how the artifacts respond to unexpected behavior. Balance system-level checks and controls and individual autonomy. Allow for information to be stored and processed to increase the overall resilience of the system. Design influence, do not design control.

\subsubsection{Consistent}

Artifacts Should Be Structurally Consistent or Coherent Consistency (Lidwell et al. 2010; Resmini and Rosati 2011) occurs at the structural, behavioral, functional, and esthetic level. Structural consistency can be seamlessly implemented across different media using different functional, behavioral, and esthetic means. It provides cognitive continuity regardless of context, device, or interface.

Heuristics Design Internet of Things artifacts with structural consistency in mind. Design a solid information architecture and then translate it behaviorally, functionally, and esthetically as needed, across as many mediums as necessary using their specialized verbal, visual, aural, and tactile vocabularies. Consistency works at different scales.

\subsubsection{Conversational}

Information flows in an ecosystem are conversations that lead to actions Communications between people and artifacts and among artifacts are conversations and negotiations. Conversations require a language for mutual understanding (Winograd and Flores 
1987). Successful conversations are part of the process of creating shared states that can lead to action: "every speech is an act; every act is a transformation; transformation acts are a set of events applied to a particular state, by subject, in order to provoke changes in future states" (Lima-Marques 2011).

Heuristics Design Internet of Things artifacts paying specific attention to interoperability and systemic information flows. Support the transformation of perceptual and linguistic information into conversations. If necessary, provide ways to access past conversations. Provide continuous and stateful non-intrusive feedback about what is happening, what will happen, and what needs to be done. Actively shape the language that will be spoken between actors and artifacts, and consider how conversations translate to visual, auditory, tactile, or gestural vocabularies. Provide fallbacks for dealing with unexpected situations.

\subsubsection{Ontological}

Design has systemic effects that change the context in which design artifacts themselves operate Design materializes human desire (Pepperel and Punt 2000). It actively transforms reality, human action, and human interpretation by bringing things into the world (Willis 1999). The action is a process endowed with a purpose in which agents, by changing something, change themselves: "products and effects are the cause and the producer of what is produced" (Morin 1992).

Heuristics Design Internet of Things artifacts for change, increased resilience, and emergence, and not for equilibrium. Stability is a dangerous illusion. Systems are constantly on the verge of collapse: homeostasis is a dynamic process of constant change. Design for the imprecise whole, not for the precise parts: "buildings should imitate ecological systems" (Yeang 2007) because "nature, not the machine, is the most important model for architecture" (A. Aalto, in Filler 2007, p. 92).

\section{Conclusions}

We posit that the Internet of Things is an instance or subset of the infosphere (Floridi 2010) and both an objective or formal superset and a subjective or contextual subset of the conceptualization of digital/physical information ecosystems as described in current information architecture theory and practice (Resmini and Rosati 2011; Resmini and Lacerda 2016).

We adopt Van Gigch and Pipino's (1986) M3 Metamodel Methodology to clarify the dynamics between design practice and design theory in the field of information architecture. We describe how information architecture has preserved its epistemological purpose but has nonetheless seen its paradigm layer change under the influence of socio-technical changes that have redefined how we conceptualize information spaces. We articulate how this reframing is yet to be fully addressed in both the science (theory) and practice (solutions) layers. 
We argue that the purpose of the socio-technical change is the transformation of human imagination and desire into agency and potential for action in the world (Pepperel and Punt 2000) and that social, cultural, esthetic, and ethical values are primary objectives to design together with technological, organizational, and commercial values. To this extent, and with the goal of supporting reflective practices in information architecture that can restart the necessary conversation between "techne" and "episteme," we propose a pervasive information architecture framework for designing Internet of Things artifacts based on a phenomenological human-centered approach.

This framework considers artifacts systemically as elements of information-based cross-channel ecosystems and introduces a categorized set of 16 medium-independent principles or heuristics in the tradition of principle-based design that target: the human perspective, pertaining to the relationship between agents and artifacts; the architectural perspective, pertaining to the artifacts themselves; and the systemic perspective, pertaining to ecosystem-level dynamics. We submit these principles for further discussion and verification, to validate its application for cross-channel information ecosystems in general and for Internet of Things artifacts in particular.

Open Access This article is distributed under the terms of the Creative Commons Attribution 4.0 International License (http://creativecommons.org/licenses/by/4.0/), which permits unrestricted use, distribution, and reproduction in any medium, provided you give appropriate credit to the original author(s) and the source, provide a link to the Creative Commons license, and indicate if changes were made.

Publisher's Note Springer Nature remains neutral with regard to jurisdictional claims in published maps and institutional affiliations.

\section{References}

Arthur, P., \& Passini, R. (1992). Wayfinding: People, Signs, and Architecture. New York: McGraw-Hill.

Ashton, K. (2009). That 'Internet of Things' thing. RFID Journal. http://www.rfidjournal. com/articles/view?4986.

Benyon, D. (2014). Spaces of Interaction, Places for Experience. Morgan \& Claypool. http://ieeexplore.ieee. org/xpl/articleDetails.jsp?arnumber=6920433. Accessed 19 Nov 2015.

Benyon, D., \& Resmini, A. (2017). User experience in cross-channel ecosystems. In BCS learning and development. Proceedings of British HCI 2017 - digital make-believe. (p. 12). British HCI 2017. http://hci2017.bcs.org/wp-content/uploads/33.pdf.

Castells, M. (2002). The Internet Galaxy: Reflections on the Internet, Business, and Society. Oxford: Oxford University Press.

Chalmers, M., Dieberger, A., Höök, K., \& Rudström, Å. (2004). Social navigation and seamful design. Japanese Journal of Cognitive Science, Special Issue on Social Navigation, 11(3), 171-181.

Conti, M., Das, S. K., Bisdikianc, C., Kumar, M., Ni, L. M., Passarella, A., Roussos, G., Tröster, G., Tsudikg, G., \& Zambonelli, F. (2012). Looking ahead in pervasive computing: challenges and opportunities in the era of cyber-physical convergence. Pervasive and Mobile Computing., 8(1), 2-21.

Dourish, P. (2004). Where the Action is: The Foundations of Embodied Interaction. Cambridge: The MIT Press.

Filler, M. (2007). Makers of Modern Architecture. Volume I. New York: New York Review Books.

Floridi, L. (2010). Information: A Very Short Introduction. Oxford: Oxford University Press.

Floridi, L. (2014). The Fourth Revolution: How the Infosphere is Reshaping Human Reality. Oxford: Oxford University Press. 
Foltz, M. A. (1998). Designing Navigable Information Spaces. Master of Science, Massachusetts Institute of Technology, Department of Electrical Engineering and Computer Science.

Gartner (2017). Gartner says 8.4 billion connected "things" will be in use in 2017, Up 31 Percent From 2016. https://www.gartner.com/newsroom/id/3598917. Accessed 20 April 2017.

Gibson, J. J. (1979). The Ecological approach to visual perception. New Jersey: Lawrence Erlbaum Associates.

Van Gigch, J. P., \& Pipino, L. L. (1986). In search of a paradigm for the discipline of information systems. Future Computing Systems, 1(1), 71-97.

Greenfield, A. (2006). Everyware: The Dawning Age of Ubiquitous Computing. San Francisco: New Riders.

Harrison, S., and Dourish, P. (1996). Re-place-ing space: the roles of place and space in collaborative systems. Proceedings of the 1996 ACM conference on Computer supported cooperative work. ACM. Pp. 67-76). Doi:https://doi.org/10.1145/240080.240193.

Hinton, A. (2014). Understanding Context: Environment, Language, and Information Architecture. Sebastopol: O'Reilly.

Husserl, E. (1931). Ideas: General Introdution to Pure Phenomenology (1st ed.). New York: Macmillan Company.

Information Architecture Institute (2016). What is information architecture. https://www.iainstitute.org/what-is-ia. Accessed 20 April 2017.

Ishii, H. (2012). Defy gravity: beyond tangible bits, toward radical atoms. Proceedings of DW/AD12. http://www.youtube.com/watch?v=SMdFus2nPyw. Accessed 20 Sept 2017.

Kazmierczak, E. (2003). Design as meaning making: from making things to the design of thinking. Design Issues, 19(2), 45-59.

Kirby, A. (2009). Digimodernism: How New Technologies Dismantle the Postmodern and Reconfigure Our Culture. London: Continuum.

Kopetz, H. (2011). Real-Time Systems: Design Principles for Distributed Embedded Applications. Real-Time Systems Series. Boston: Springer. https://doi.org/10.1007/978-1-4419-8237-7.

Kuniavsky, M. (2010). Smart Things. Burlington: Morgan Kaufmann.

Lacerda, Flávia (2015). Arquitetura da Informação Pervasiva: projetos de ecossistemas de informação na Internet das Coisas. Doctoral dissertation. Brasília: Universidade de Brasília. 226 fl. M. Lima-Marques, supervisor. A. Resmini, sec. supervisor. http://repositorio.unb.br/bitstream/10482/19646/1/2015_ FlaviaLacerda.pdf. Accessed 09 Sept 2017.

Lacerda, F., \& Lima-Marques, M. (2014). Information Architecture as a Discipline: a Methodological Approach. In A. Resmini (Ed.), Reframing Information Architecture. Human-Computer Interactions Series. Cham (pp. 1-10). Springer.

Latour, B. (1996). On actor-network theory: a few clarifications plus more than a few complications. Philosophia, 25(3), 47-64.

Lidwell, W., Holden, K., \& Butler, J. (2010). Universal Principles of Design: 125 Ways to Enhance Usability, Influence Perception, Increase Appeal, Make Better Design Decisions, and Teach Through Design. Beverly: Rockport Publishers.

Lima-Marques, M. (2011). Outline of a theoretical framework of Architecture of Information: a School of Brasilia proposal. In J. Y. Beziau \& M. E. Coniglio (Eds.), Logic without Frontiers. Festschrift for Walter Alexandre Carnielli on the occasion of his 60th birthday. London: College Publications.

Lucas, P., Ballay, J., \& McManus, M. (2012). Trillions: Thriving in the Emerging Information Ecology. New Jersey: Wiley.

Lynch, K. (1960). The image of the city. Cambridge: The MIT Press.

Maeda, J. (2006). The Laws of Simplicity. Cambridge: The MIT Press.

McCullough, M. (2004). Digital Ground: Architecture, Pervasive Computing, And Environmental Knowing. Cambridge: The MIT Press.

McEwen, A., \& Cassimally, H. (2013). Designing the Internet of Things. New Jersey: Wiley.

McLuhan, M. (1965). Understanding Media: The Extensions of Man. New York: McGraw-Hill.

Meadows, D. H. (2008). Thinking in systems: a primer. (D. Wright, Ed.). Vermont: Chelsea Green Pub.

Merleau-Ponty, M. (1963). The Structure of Behavior. Boston: Beacon Press.

Mitchell, W. J. (2006). Smart City 2020. Metropolis. http://www.metropolismag.com/uncategorized/smart-city-2020/.

Mitew, T (2008). The politics of networks using actor network theory to trace techniques collectives and space times. Sydney: Curtin University of Technology. $\mathrm{PhD}$ Thesis.

Morin, E. (1992). From the concept of system to the paradigm of complexity. Journal of Social and Evolutionary Systems, 15(4), 371-385. https://doi.org/10.1016/1061-7361(92)90024-8.

Norberg-Schulz, C. (1971). Existence, Space and Architecture. Westport: Praeger Publishers.

Norman, D. (2009). The Design Of Future Things. New York: Basic Books. 
Norris, S. (2013). Cross-channel design: a primer. https:/www.nomensa.com/blog/2013/cross-channeldesign-a-primer. Accessed 20 Sept 2017.

Pepperel, R., \& Punt, M. (2000). The Postdigital Membrane: Imagination, Technology, and Desire. Wilmington: Intellect Books.

Resmini, A. (2013). The architectures of information (Les architectures d'information). Études de Communication. Vol. 41. http://edc.revues.org/5380. Accessed 20 Sept 2017.

Resmini, A. (2014). Preface. In A. Resmini (Ed.), Reframing Information Architecture. Human-Computer Interactions Series. Cham: Springer.

Resmini, A., \& Lacerda, F. (2016). The architecture of cross-channel ecosystems: from convergence to experience. Proceedings of the 8th International Conference on Management of Digital EcoSystems. Pp. 17-21. ACM. http://dl.acm.org/citation.cfm?id=3012087. Accessed 09 Sept 2017.

Resmini, A., and Rosati, L. (2009). Information architecture for ubiquitous ecologies. Proceedings of the International Conference on Management of Emergent Digital EcoSystems MEDES'09. Pp. 196-199). ACM. Doi:https://doi.org/10.1145/1643823.1643859.

Resmini, A., \& Rosati, L. (2011). Pervasive information architecture: Designing cross-channel user experiences. Burlington: Morgan Kaufmann.

Rose, D. (2014). Enchanted Objects. New York: Scribner Book Company.

Rosenfeld, L., \& Morville, P. (1998). Information Architecture for the World Wide Web (1st ed.). Sebastopol: O'Reilly.

Rosenfeld, L., \& Morville, P. (2006). Information Architecture for the World Wide Web (3rd ed.). Sebastopol: O'Reilly.

Sheppard, R.Z. (1971). Rock candy. Time Magazine, Apr. 12, 1971. http://content.time. com/time/magazine/article/0,9171,905004,00.html

Tate, T. (2011). The rise of cross-channel UX design. UX Matters. http://www.uxmatters. com/mt/archives/2011/10/the-rise-of-cross-channel-uX-design.php. Accessed 20 Sept 2017.

Thelen, E., Schöner, G., Scheier, C., \& Smith, L. B. (2001). The dynamics of embodiment: a field theory of infant perseverative reaching. Behavioral and Brain Sciences, 24(01), 1-34. https://doi.org/10.1017 /S0140525X01003910.

Toffler, A. (1980). The Third Wave. New York: Bantam Books.

Tuan, Y. F. (1977). Space and place: the perspective of experience. University of Minnesota Press.

Varela, F. J., Thompson, E., \& Rosch, E. (1993). The Embodied Mind: Cognitive Science and Human Experience. Cambridge: The MIT Press.

Vitruvius Pollio, M. (1999). In I. D. Rowland \& T. N. Howe (Eds.), Ten Books on Architecture (De Architectura). Cambridge: Cambridge University Press.

von Bertalanffy, L. (1998). General system theory (Revised ed.). New York: George Braziller, Inc.

Weiser, M. (1999). The computer for the 21st century. ACM SIGMOBILE Mobile Computing and Communications Review - Special issue dedicated to Mark Weiser., 3(3), 3-11.

Willis, A. M. (1999). Ontological Designing. Proceedings of Design Cultures 1999, Conference of the European Academy of Design, Sheffield Hallam University.

Winograd, T., \& Flores, F. (1987). Understanding Computers and Cognition. Boston: Addison-Wesley.

Wurman, R. S. (1997). Information Architects. New York: Graphis Inc.

Yeang, K. (2007). Q\&a: Ken Yeang interview. CNN. http://edition.cnn.com/2007/TECH/science/07/16 /yeang.qa/. Accessed 19 Sept 2017.

\section{Affiliations}

\section{Flávia Lacerda ${ }^{1} \cdot$ Mamede Lima-Marques $^{2} \cdot$ Andrea Resmini $^{3}$}

Mamede Lima-Marques

mamede@modal.org.br

1 Brazilian Federal Court of Accounts (TCU), Serzedello Correa Institute (ISC), Brasília, Brazil

2 The Modal Institute of Science, Technology and Innovation, Brasília, Brazil

3 Jönköping University, Jönköping, Sweden 